1991 : mise en place des institutions pionnières (l'école d'agriculture); 1911-1962 : rayonnement des institutions agronomiques; 1962-1995 : diversification économique; 1995-2005 redéploiement et complexification des éléments du système d'innovation. On aura compris que cette dernière phase se prolongera pendant encore un bon nombre d'années. On imagine bien que derrière toutes ses institutions il y a des hommes et pas toujours avec des femmes derrière eux car certains sont de bons curés comme l'abbé Joseph Richard, directeur de l'école d'agriculture qui, au tournant du siècle (le xxè), a implanté une méthode de labour qui portera son nom et qui deviendra une norme culturale dans la vallée du StLaurent.

En faisant un saut dans le temps et dans le volume, on en arrive au contrat du siècle: Bombardier en 1982 a obtenu une commande pour la fabrication de plus de 800 voitures pour le métro de New York. On comprendra que cette impulsion donnée au secteur privé a eu pour conséquence de modifier profon-dément la structure économique de La Pocatière. On assista à l'avènement d'un contexte favorisant des collaborations inédites entre une partie du dispositif institutionnel du système local d'innovation et le secteur privé. Moins glorieux, cependant, est ce contrat obtenu en 2007, sans recours à un appel d'offre, afin de renouveler la flotte du métro de Montréal suite à une forte mobilisation l'année précédente de la population locale. La politique a ses raisons que la raison ne saurait comprendre...

L'ouvrage se termine par le chapitre 5 dont l'objet est d'analyser le fonctionnement $\mathrm{du}$ système local d'innovation. L'effort de généralisation des auteurs s'avère ici un élément important de leur contribution car tout lecteur appartenant à une région non métropolitaine tirera profit des éléments mis ici en évidence. Ce lecteur est appelé à vérifier si, dans sa région, à l'instar de ce qui s'observe à La Pocatière, le système local d'innovation perdure non pas à cause d'une dynamique de coordination interentreprises mais en vertu d'une dynamique de coopération interinsti-tutionnelle. Car c'est bien ce qui se dégage à la fin de ce long voyage dans le temps : le système d'innovation de la Pocatière a comme caractéristique fondamentale un cadre institutionnel à la fois fort et prépondérant qui favorise trois types de collaboration: les collaborations interinstitutionnelles il va sans dire; les collaborations interentreprises (essentiellement à l'extérieur du milieu, les collaborations locales étant pratiquement inexistantes); les collaborations entre les entreprises et les institutions.

En s'appuyant sur les données secondaires disponibles et sur des entrevues de type non-structurées auprès d'acteurs locaux, les auteurs sont parvenus à bien mettre en évidence les éléments susceptibles de donner à une région non métropolitaine un dynamisme durable. Vivement des études similaires appliquées à d'autres milieux.

* Messieurs les auteurs! Que l'on parle de pôles, de champs ou de secteurs d'activité, le mot «activité» ne prend pas de «s». Que le directeur d'O\&T en prenne bonne note également...

\section{Coalition pour un Québec des régions, Libérer les QuébecS : décentralisation et démocratie, Montréal, Écosociété,176 p.}

On sait que l'expression Deux Québec dans un a fait florès, or, avec ce nouvel ouvrage sur la décentralisation, parlera-t-on de Dix-sept Québec dans $u n$ ? Plus de pouvoir aux régions, ce vœu, qui n'a rien de nouveau, est repris ici par une coalition présentée comme un regroupement de personnalités, engagées dans la réforme démocratique et le développement régional, qui revendique la mise en place de véritables gouvernements territoriaux imputables et autonomes, et - tant qu'à y être - la réforme de nos institutions démocratiques. Le directeur d' $O \& T$ fait partie de cet aréopage placé sous la houlette du médiatique Roméo Bouchard (R.B.) qui n'en n'est pas à son premier fait d'armes en relation avec la problématique de la décentralisation puisque dans ces pages, j'ai fait la recension de son $Y$-a-t-il un avenir pour les régions? ${ }^{l} \mathrm{Si}$ l'ex-président de l'Union paysanne se trouve au cœur de cet ouvrage, il a obtenu la collaboration de quelques membres de la coalition dont certains sont familiers aux lecteurs d' $O \& T$.

Dans un premier chapitre, celui qui s'est d'abord fait connaître comme étant le pourfendeur du productivisme agricole à tout crin, se veut très ambitieux; il se fait le prêche de rien de moins qu'une deuxième Révolution tranquille. Une révolution qui prendrait la forme d'une réappropriation du territoire et d'une gouvernance par les citoyens conformément au rêve inachevé (ce n'est pas le seul) de René Lévesque. En fait, il s'agirait d'une deuxième phase de la Révolution tranquille. Un enthousiasme quelque peu débridé conduit R.B. à écrire: «Après s'être doté d'un État fort (dans un Canada uni ${ }^{2}$ ) le moment est venu de nous doter d'un pays réel, d'un pays qui appartient à ses concitoyens d'un bout à l'autre de notre territoire, d'un pays tourné vers le monde.» $\mathrm{Ne}$ manque que la date du prochain référendum... 
Dans le chapitre 2, R.B. s'aventure à suggérer des champs d'action dont les régions pourraient avantageusement assumer la responsabilité :

- Implantation et gestion des équipements culturels, récréatifs et de support aux activités industrielles ou commerciales;

- L'éduction préscolaire, primaire et secondaire (en espérant que l'on ne se retrouvera pas avec 18 ministères de l'Éducation);

- La santé de première ligne et les politiques sociales (même espoir que pour le point précédent...).

Évidemment, R.B. suppose que les ressources fiscales et financières requises vont suivre: pas de pelletage de responsabilités sans fournir la pelle.

Mais notre leader régional fait preuve de lucidité : yen n'aura pas de facile comme dirait Michel Bergeron à $110 \%$. En effet, il reconnaît l'existence d'une certaine résistance et une tout aussi certaine inertie face à la question de la décentralisation, à la fois parmi la population (qui a besoin du grand frère Québec) et les élus locaux (enveloppés dans leurs habitudes). Mais, il a un argument massue : les convictions (ou les rêves) de René Lévesque. Cette référence lui apparaît tellement précieuse qu'il cite à deux reprises (p. 8 et 29) le même passage du Livre blanc sur la décentralisation, publié en 1977. D'autres passages de ce document demeuré célèbre sont offerts aux lecteurs. Il en va ainsi pour un texte de G. Courtemanche qui se voit cité à quelques reprises, et ce avec beaucoup de pertinence, puisque dans la recension de l'ouvrage publié par Solidarité rurale du Québec: Pour une décentralisation démocratique, j'avais mentionné que son chapitre était l'une des surprises agréables de ce volume ${ }^{3}$.

Pour le chapitre 3 , de toute évidence rédigé de sa propre main, RB, a cru utile d'ajouter les noms de l'éditeur d' $O \& T$ et d'André Laroque. On peut y lire que dans un pays (oui, c'est bien écrit pays...) étendu et diversifié comme le Québec, il est essentiel de ne pas appliquer la décentralisation territoriale d'une façon uniforme dans le temps et dans l'espace. Le transfert de juridictions devra se faire en lien avec les instances locales et régionales, selon les besoins et les capacités de chaque territoire selon un menu ouvert et d'un agenda à la pièce. Ouf! Après l'échec du fédéralisme asymétrique, allons-nous avoir une décentralisation asymétrique réussie? Je suggère à R.B.d'adhérer à Québec Solidaire (si ce n'est déjà fait) et de travailler à inclure ce plan dans son programme électoral (qu'en penseraient Françoise et Amir?) car ce ne sont pas le PLQ, le PQ et pas davantage l'ADQ, ce parti des régions (!) qui vont s'engager dans une telle aventure. Le chapitre 4, intitulé L'ancrage des gouvernements territoriaux, comprend des contributions de plusieurs auteurs. La première, sous la plume d'Yvon Leclerc aujourd'hui rédacteur en chef de Réseaux, a été publiée telle quel dans le numéro d'O\&T sur La nouvelle ruralité territorialisée ${ }^{4}$. Avec André Laroque, ancien conseiller de René Lévesque, c'est bien le Dix-sept Québec dans un qui se voit préconisé puisque: ...« nos régions administratives actuelles ont toutes la consistance qu'il faut pour devenir des régions politiques.» $\mathrm{Si}$ on a connu des fusions (et des défusions) municipales, on ne devrait pas connaître des fusions régionales. Le Centre-du-Québec autrefois rattaché sous le nom de Bois-Francs à la Mauricie, conservera son intégrité territoriale. Mais notre politologue, s'il prend le soin d'écrire le mot «pays » entre guillemets, ne cache pas un certain nombre de questions, soit sept exactement, toutes formulées de la même façon : une fois établie, une fois assurée, une fois assumée... Autant de questions qui se veulent des suggestions de prérogatives régionales. Qu'on en juge: les universités et les cegeps, les hôpitaux et autres CLSC, la culture (vivement d'autres Cirque du Soleil ou Héloïse), le développement économique, le tourisme, l'accueil des immigrants. Non, il ne recommande pas de transformer l'édifice au bout de la Grande Allée en musée...Il ne va pas jusque là.

L'ouvrage est agrémenté de témoignages d'élus locaux, tel celui du préfet de la MRC du Granit (qui a au sud le Maine pour voisin) dont la réflexion sur le sujet n'est pas aussi avancée que d'autres collaborateurs à ce volume puisqu'il se demande, face à la décentralisation, quel palier choisir. La municipalité, la MRC, la région administrative ou un mélange de tout cela? Amoureux de la poutine, les Québécois pourraient être sensibles au mélange en question. Le tout se termine par une enfilade d'annexes dont la première se rapporte au livre Blanc de René Lévesque qu'affectionne tant R. B. Un texte de la Fédération québécoise des municipalités me paraît plus pertinent étant donné la nature du débat actuel. On y trouve huit pistes de décentralisation identifiées par les préfets et les directeurs généraux de MRC. Elles vont de la gestion des ressources naturelles à celle des équipements touristiques de loisir et culturels en passant par celle des réseaux routiers et du transport collectif. Autant de propositions réalistes qui ne semblent pas devoir faire appel à la création d'une panoplie de nouveaux ministères...

Au temps de la télévision en noir et blanc, à l'été 1965, j'ai occupé un emploi d'étudiant au ministère de l'Agriculture. J'avais pour patron le seul économiste du ministère. Lors d'une discussion sur le découpage administratif (le Québec avait alors dix régions) il avait affirmé que ce découpage était purement arbitraire : $L a$ où il y a du monde avait-il simplement dit suite à mon questionnement. La décentralisation souhaitée par cette 
coalition ou par toutes autres personnes (elles sont nombreuses) exigerait sûrement un autre découpage que celui que l'on connaît. Le prochain ouvrage sur la décentralisation devra se pencher sur cette nécessité, sinon, moi, je refuserai d'en faire la recension.

${ }^{1}$ Volume 16, No 1, hiver 2007

${ }^{2}$ Note de l'auteur.

${ }^{3}$ Volume 16, No 1, hiver 2007

${ }^{4}$ Volume 17 , no 1 , hiver 2008

\section{Pierre Merlin, L'aménagement $d u$ territoire en France, Paris, La Documentation française, 2007, 174 p.}

L'expression « aménagement du territoire (AduT) » se veut typiquement française même si les Québécois l'ont importée quelques années avant la création du Bureau d'aménagement de l'Est du Québec (le trop célèbre BAEQ dont il ne faut pas rappeler à la mémoire des Gaspésiens). Les Wallons refusent de l'employer et les Suisses, à ma connaissance, ne l'emploient guère. Reste, bien sûr, l'Afrique francophone qui ne se prive pas de l'utiliser à défaut d'aménager véritablement. L'expression a été popularisée avec la création de la Délégation à l'aménagement et à l'action régionale (DATAR) en 1963 avec Olivier Guichard, grand gaulliste devant l'éternel, qui en fut le tout premier délégué. Il s'agissait alors de conseiller l'État dans sa volonté de favoriser, à travers les fameux plans quinquennaux, un développement harmonieux de l'Hexagone en cherchant à décongestionner Paris et à favoriser un meilleur équilibre entre les régions. Amenuiser les disparités régionales, voilà l'idéal que reprendra à sa création, en 1969, notre cher Office de planification et de développement du Québec qui, en fait, ne planifiera guère tout en ne développant pas davantage. Mais ne dit-on pas que c'est l'intention qui compte? En ce qui regarde l'AduT, le concept faisait déjà partie des préoccupations du Conseil d'orientation économique, sous l'égide de Roland Parenteau, avant l'avènement de l'OPDQ. Le concept fut rapatrié à la faveur du retour de France de jeunes universitaires engagés sur les traces de leurs professeurs ayant obtenus leurs galons en France dans les années d'après guerre. Ces derniers avaient été recrutés par le père Lévesque, fondateur de la Faculté des sciences sociales au grand dam d'un certain premier ministre de l'époque car, contrairement aux évêques, lui, il refusait de manger dans sa main. En 1964, à titre de finissant d'une formation de premier cycle à l'Université Laval, l'AduT avait été le thème de mon séminaire de fin d'étude. À la bibliothèque, $\mathrm{j}$ 'avais pu trouver quatre ou cinq références... Cet ouvrage de
Pierre Merlin, professeur émérite de l'Université de Paris I, montre, ô combien, il s'en est coulé de l'eau sous le pont Neuf ou sous celui de Québec depuis lors. Car les références sur le sujet, aujourd'hui, comme les feuilles d'automne, se ramassent à la pelle ou presque.

L'auteur montre, en fait, que la notion d'AduT, a fait son apparition au lendemain de la guerre (la deuxième, bien sûr) alors qu'il fallait reconstruire l'Europe. Elle est devenue, comme il l'écrit, une priorité de l'action de l'État dans les années 1960 sous l'influence du volontariste gaulliste. Suite à une recension portant sur la décentralisation ${ }^{1}$, il est intéressant de voir comment en France s'est présenté le conflit centralisation versus décentralisation. Cette dernière remportera une bataille avec les lois Deferre 1982-83. Mais les partisans des Jacobins ne lâchant jamais prise, les disciples des Girondins ne sont jamais assurés de remporter une guerre qui n'en finit jamais de finir. L'évolution des différentes politiques françaises ne peut manquer d'intéresser tous ceux qui, au Québec, appellent de tous leurs vœux des réformes susceptibles de conduire à une diminution de l'emprise de Québec sur le sort des régions. Celui qui a écrit pas moins d'une cinquantaine d'ouvrages sur des sujets entourant la problématique de l'AduT, dégage un constat pessimiste en ce qui regarde la situation prévalant en France. À plusieurs titres, ose-til écrire, il est tenté de penser que les évolutions observées sur une période couvrant plus de quatre décennies, comme étant des régressions. Pas facile en effet, de décentraliser, surtout un pays comme la France dont le mal principal est justement le centralisme comme l'a signalé en son temps (1976) Alain Peyrefitte.

Mais de quoi parle-t-on vraiment? Aménager le territoire, pour l'auteur, c'est se rapporter à l'action et à la pratique de disposer avec ordre à travers l'espace d'un pays et dans une vision prospective, les hommes et leurs activités, les équipements et les moyens de communication qu'ils peuvent utiliser, en prenant en compte les contraintes naturelles, humaines et économiques, et ceci afin que les fonctions et les relations entre les hommes s'exercent de la façon la plus commode, la plus économique et la plus harmonieuse. Voilà, pas plus compliqué ${ }^{2} .$. Après s'être interrogé sì aménager un territoire relève d'une démarche scientifique, ou s'il ne s'agit pas plutôt d'une technique ou encore, comme le dit le Larousse, s'il s'agit d'une forme d'art, l'auteur préfère ramener l'AduT à son caractère d'intervention volontaire, de dimension territoriale de la planification. C'est donc une praxis, i.e. un ensemble d'actions visant un même objectif. Ceci étant admis, l'auteur s'interroge sur les fondements théoriques de l'AduT. Quel en est le contenu, doit-il être global ou peut-il être thématique? 